\title{
FUNCTIONS DEFINED BY SEQUENCES OF INTEGRALS AND THE INVERSION OF APPROXIMATE DERIVED NUMBERS*
}

\author{
BY \\ R. L. JEFFERY
}

1. Introduction. Let the function $f(x)$ be measurable on $(a, b)$, and let $s=s_{1}(x), s_{2}(x), \cdots$ be a sequence of summable functions tending to $f(x)$ almost everywhere. If $f(x)$ is summable and $x$ a point of $(a, b)$, conditions under which

$$
\lim _{n \rightarrow \infty} \int_{a}^{x} s_{n}(x) d x=\int_{a}^{x} f(x) d x
$$

have been determined. $\dagger$ It is easy to construct sequences for which the limit on the left side of (1) exists and is different from the right side. In the present paper necessary and sufficient conditions are obtained for the existence of this limit in terms of the sequence $s_{n}(x)$. It turns out that the limit function $F(x)$ is independent of $f(x)$. In fact if $F(x)$ is an arbitrary continuous function, there exists a sequence of summable functions $s_{n}(x)$ tending to zero everywhere, for which $\int_{a}^{x} s_{n}(x) d x$ tends to $F(x)$ everywhere. If $F_{1}(x), F_{2}(x), \cdots$ is a sequence of measurable functions, there is a sequence $s_{n}(x)$ tending to zero everywhere for which almost everywhere the set of limits of $\int_{a}^{x} s_{n}(x) d x$ is the sequence $F_{1}(x), F_{2}(x), \cdots$. If $\int_{e} s_{n}(x) d x$ is bounded in $n$ and $e, e$ any measurable subset of $(a, b)$, then $F(x)$, when it exists, is of bounded variation on $(a, b)$. Conversely, if $F(x)$ is of bounded variation, there exists a function $f(x)$ and a sequence of summable functions $s_{n}(x)$ tending everywhere to $f(x)$ for which $\int_{a}^{x} s_{n}(x) d x$ tends to $F(x)$, and for which $\int_{e} s_{n} d x$ is bounded in $n$ and $e$. This is of some interest for the reason that it provides a characterization of functions of bounded variation which can be extended to functions of any number of variables.

It is possible for the limit of the left side of (1) to exist when the function $f(x)$ is not summable. As an aid in the study of this situation we introduce the following conventions:

* Presented to the Society, December 27, 1934, and April 20, 1935; received by the editors May 3,1935 , and in revised form November 13, 1935, and July 11, 1936.

$\dagger \mathrm{W}$. H. Young, Term by term integration of oscillating series, Proceedings of the London Mathematical Society, ser. 2, vol. 8, pp. 99-116. Jeffery, The integrability of a sequence of functions, these Transactions, vol. 33 (1931), pp. 433-440. T. H. Hildebrandt, On the interchange of limit and Lebesgue integral for a sequence of functions, ibid., pp. 441-443. In the second of these papers further references are given to the literature on the subject. 
Let $f(x)$ be measurable on $(a, b)$. If there exists a sequence of summable functions $s_{n}(x)$ tending to $f(x)$ almost everywhere and a continuous function $F(x)$ such that $\int_{a}^{x} s_{n}(x) d x$ tends to $F(x)$, then $f(x)$ is integrable in the sequence sense to $F(x), F(x)=S(f, a, x)$. If $s_{n}(x)$ can be so determined that $s_{n}(x)=f$ on $E_{n}, s_{n}(x)=0$ elsewhere, $E_{n}$ contains $E_{n-1}$, and $m E_{n}$ tends to $b-a$, then $f(x)$ is totally integrable in the sequence sense to $F(x), F(x)=T S(f, a, x)$.

In the light of the foregoing statements concerning the limits of $\int_{a}^{x} s_{n} d x$ with $s_{n}$ tending to zero, it is clear that $S(f, a, x)$ is not uniquely defined. If $f$ is finite almost everywhere, and almost everywhere is the approximate derivative of the continuous function $F(x)$, then $S(f, a, x)$ exists for which $S(f, a, x)=F(x)-F(a)$. If $f(x)$ is summable, then $T S(f, a, x)$ is uniquely defined, and $T S(f, a, x)=\int_{a}^{x} f(x) d x$. If $f(x)$ is integrable in the generalized Denjoy sense, and $F(x)=\int_{a}^{x} f(x) d x$, then there exists $T S(f, a, x)=F(x)$, but in this case $T S(f, a, x)$ is not uniquely defined. It is probable that if $f(x)$ is integrable in the generalized Denjoy sense and $T S(f, a, x)$ can be determined which is $(A C G),{ }^{*}$ then $T S(f, a, x)=F(x)$. Some information is given in regard to this point, but so far it has not been possible to obtain all the facts.

2. The limit function of the sequence of integrals. Let $f$ be summable, and let $s_{n}$ be a sequence of summable functions tending to $f$ almost everywhere. For an arbitrary positive number $\eta$ let $E(l, \eta)$ be the part of $(a, x)$ for which $\left|f-s_{n}\right|<\eta, n \geqq l$, and let $C(l, \eta)$ be the complement of $E(l, \eta)$ on $(a, x)$. These sets are measurable, and as $l$ increases $m C(l, \eta)$ tends to zero. If for a fixed $x$

$$
F(x)=\lim _{n \rightarrow \infty} \int_{a}^{x} s_{n} d x=\lim _{n \rightarrow \infty} \int_{E(l, \eta)} s_{n} d x+\lim _{n \rightarrow \infty} \int_{C(l, \eta)} s_{n} d x
$$

then for $l$ sufficiently large the first limit on the right is arbitrarily near to $\int_{a}^{x} f d x$. Hence if $F(x)$ exists, we have

$$
F(x)=\int_{a}^{x} f d x+\lim _{l \rightarrow \infty}\left[\lim _{n \rightarrow \infty} \int_{c(l, \eta)} s_{n} d x\right] .
$$

We thus get:

THEOREM I. A necessary and sufficient condition for the existence of $F(x)$ is the existence of

$$
\lim _{l \rightarrow \infty} \int_{C(l, \eta)} s_{n} d x
$$
$n \geqq l$.

* Generalized absolutely continuous. $F(x)$ is $(A C G)$ on $(a, b)$ if it is continuous, and $(a, b)$ can be separated into a finite or denumerable set of sets $E_{1}, E_{2}, \cdots$ such that $F(x)$ is absolutely continuous on each $E_{n}$; Saks, Thérie de l'Intégral, Warsaw, 1933, p. $152, \$ 9$. 
Let $U(n, \delta), L(n, \delta)$ be respectively the least upper bound and greatest lower bound of $\int_{e} s_{n}$ for all $e$ on $(a, x)$ with $m e<\delta$. The reasoning used by Hildebrandt* can be modified to give:

THEOREM II. A necessary and sufficient condition that $F(x)$ exists is that

$$
\lim _{\delta \rightarrow 0} \lim _{n \rightarrow \infty}[U(n, \delta)+L(n, \delta)]=K(x) .
$$

Let $g=g_{1}, g_{2}, \cdots$ be any subsequence of $s$, and let $U(g, n, \delta), L(g, n, \delta)$ be the least upper bound and greatest lower bound respectively of $\int_{e} g_{i}, m e<\delta$, $i=1,2, \cdots, n$.

Theorem III. A necessary and sufficient condition that $F(x)$ exist is that $\lim _{\delta \rightarrow 0} \lim _{n \rightarrow \infty}[U(g, n, \delta)+L(g, n, \delta)]=\bar{K}$,

where $K$ depends on $x$ but is independent of $g$.

The proof of this can be accomplished by the methods of our previous paper. $\dagger$ We give here a proof which is much simpler and which includes the results of that paper as a special cáse.

Suppose $F(x)$ exists. Then by Theorem I of the present paper we have

$$
\lim _{l \rightarrow \infty} \int_{c(l, \eta)} s_{n} d x=K, \quad n \geqq l .
$$

Suppose there is some $g$ of $s$ such that for every $\delta^{\prime}>0$ there exists $\delta<\delta^{\prime}$ and a sequence of positive integers $n_{1}, n_{2}, \cdots$ : such that

$$
U\left(g, n_{i}, \delta\right)+L\left(g, n_{i}, \delta\right)>K+\lambda, \quad \lambda>0 .
$$

Fix $l$ so that

$$
\left|\int_{C(l, \eta)} s_{n} d x-K\right|<\frac{\lambda}{4}, \quad n \geqq l .
$$

For a fixed $n, U(g, n, \delta)$ tends to zero as $\delta$ tends to zero. As a result of this, together with (2), it follows that there exists $\delta, n_{i}, n_{i}^{\prime}$, and a set $e$, such that $l<n_{i}^{\prime} \leqq n_{i}, m e<\delta$, and

$$
\int_{e} g_{n_{i}^{\prime}} d x>U\left(g, n_{i}, \delta\right)-\frac{\lambda}{4}
$$

If $C^{+}$and $C^{-}$are the parts of $C(l, \eta)$ for which $g_{n_{i}^{\prime}} \geqq 0, g_{n_{i}^{\prime}}<0$, respectively, reasoning similar to that used by Hildebrandt $\ddagger$ shows that

* Loc. cit., pp. 441-442.

$\dagger$ These Transactions, loc. cit.

$\ddagger$ Loc. cit., p. 442, lines 1-7. 


$$
\int_{c^{+}} g_{n_{i}} d x>U\left(g, n_{i}, \delta\right)-\frac{\lambda}{2},
$$

while the definition of $L(g, n, \delta)$ gives

$$
\int_{C^{-}} g_{n_{i}^{\prime}} d x \geqq L\left(g, n_{i}, \delta\right) .
$$

Hence, since $C^{+}+C^{-}=C(l, \eta)$,

$$
\int_{C(l, \eta)} g_{n_{i}^{\prime}} d x>U\left(g, n_{i}, \delta\right)+L\left(g, n_{i}, \delta\right)-\frac{\lambda}{2}>K+\frac{\lambda}{2} .
$$

Since $n_{i}^{\prime}>l$ this contradicts (3). Similar reasoning leads to a contradiction, if in (2) $K+\lambda$ is replaced by $K-\lambda$ and the inequality sign is reversed. This shows that the condition is necessary.

Next suppose that the condition holds and $F(x)$ does not exist. If there exists a subsequence $g=g_{1}, g_{2}, \cdots$ of $s$ such that $\int_{a}^{z} g_{n} d x$ tends to $\pm \infty$, the method of the first part of the theorem can be used to show that

$$
\lim _{\delta \rightarrow 0} \lim _{n \rightarrow \infty}[U(g, n, \delta)+L(g, n, \delta)]= \pm \infty .
$$

If no subsequence exists and $F(x)$ does not exist, there then exists two subsequences $g=g_{1}, g_{2}, \ldots$ and $h=h_{1}, h_{2}, \ldots$ of $s$ such that

$$
\lim _{n \rightarrow \infty} \int_{a}^{x} g_{n} d x=G>\lim _{n \rightarrow \infty} \int_{a}^{x} h_{n} d x=H .
$$

By Theorem I,

$$
G=\int_{a}^{x} f d x+G^{\prime}, \quad H=\int_{a}^{x} f d x+H^{\prime} .
$$

Hence $G^{\prime}>H^{\prime}$. By the first part of this theorem

$$
\begin{aligned}
& \lim _{\delta \rightarrow 0} \lim _{n \rightarrow \infty}[U(g, n, \delta)+L(g, n, \delta)]=G^{\prime}, \\
& \lim _{\delta \rightarrow 0} \lim _{n \rightarrow \infty}[U(h, n, \delta)+L(h, n, \delta)]=H^{\prime} .
\end{aligned}
$$

Since $G^{\prime}>H^{\prime}$ the hypotheses are contradicted, and the sufficiency of the condition follows.

If $s_{n}$ is such that on $(a, b), \int_{e} s_{n} d x$ is bounded in $n$ and $e$, then for a fixed $x$ and $g$

$$
\lim _{\delta \rightarrow 0} \lim _{n \rightarrow \infty} U(g, n, \delta)=U(g, x), \quad \lim _{\delta \rightarrow 0} \lim _{n \rightarrow \infty} L(g, n, \delta)=L(g, x) .
$$


The necessary and sufficient condition for the existence of the function $F(x)$ then becomes

$$
U(g, x)+L(g, x)=K(x),
$$

where $K$ is independent of $g$. The functions $U(g, x)$ and $L(g, x)$ are monotone in $x$, and bounded. Consequently $K(x)$ is of bounded variation, and this shows that

$$
F(x)=\int_{a}^{x} f d x+K(x)
$$

is of bounded variation, a fact which can easily be proved independently of the foregoing. We now prove

THEOREM IV. If $F(x)$ is a function of bounded variation on $(a, b)$, then there exists a function $f(x)$ and a sequence of summable functions $s_{n}(x)$ tending to $f(x)$ with $\int_{e} s_{n}(x) d x$ bounded in $n$ and $e$ such that $\int_{a}^{x} s_{n}(x) d x$ tends to $F(x)-F(a)$.

If $d_{i}$ is a discontinuity of $F$, set

$$
\begin{gathered}
s_{l}\left(d_{i}\right)=F\left(d_{i}\right)-F\left(d_{i}-0\right), \quad s_{r}\left(d_{i}\right)=F\left(d_{i}+0\right)-F\left(d_{i}\right), \\
\phi(x)=\sum_{a \leqq d_{i<x}} s_{r}\left(d_{i}\right)+\sum_{a \leqq d_{i} \leqq x} s_{l}\left(d_{i}\right) .
\end{gathered}
$$

Then $F(x)=\phi(x)+\psi(x)$ where $\psi(x)$ is continuous. ${ }^{*}$ Hence given $\epsilon>0$ there exists $\delta>0$ such that if $x^{\prime}$ and $x^{\prime \prime}$ are any two points on $(a, b)$ with $\left|x^{\prime}-x^{\prime \prime}\right|$ $<\delta$ then

$$
\left|F\left(x^{\prime}\right)-F\left(x^{\prime \prime}\right)\right|<\sum_{(a, b)}\left|s_{l}\left(d_{i}\right)\right|+\sum_{(a, b)}\left|s_{r}\left(d_{i}\right)\right|+\epsilon .
$$

Arrange the discontinuities of $F$ in a definite order $d_{1}, d_{2}, \cdots$, and consider the intervals $A_{n}$ defined on $(a, b)$ by the points $d_{1}, \cdots, d_{n}$, where

$$
\sum_{i=n+1}^{\infty}\left|s_{l}\left(d_{i}\right)\right|+\sum_{i=n+1}^{\infty}\left|s_{r}\left(d_{i}\right)\right|<\epsilon .
$$

Let $(a, d)$ be the first of these intervals and let $F_{1}^{\prime}=F^{\prime}$, where $F^{\prime}$ is finite, $F_{i}^{\prime}=0$ elsewhere. On this interval $(a, d)$ there is a set of points $E$ with $m E=d-a$ at which (i) $F$ is continuous; (ii) $F^{\prime}$ exists; (iii) $\int_{x}^{x+h} F_{1}^{\prime} d x / h$ tends to $F^{\prime}$ as $h$ tends to zero; (iv) each point of $E$ is a point of density of $E$. From (iii) we get, for $h$ sufficiently small,

* Lebesgue, Leçons sur l'Intégration, Paris, 1928, p. 61. 


$$
\left|\frac{F(x+h)-F(x)}{h}-\frac{1}{h} \int_{x}^{x+h} F_{1}^{\prime} d x\right|<\epsilon .
$$

From this it follows that for each $x$ of $E$ there exists $\delta^{\prime}<\delta, \delta$ fixed in (1) above, for which

$$
\left|F(\xi)-F(x)-\int_{x}^{\xi} F_{1}^{\prime} d x\right|<\epsilon(\xi-x), \quad\left(x \leqq \xi \leqq x+\delta^{\prime}\right),
$$

where, on account of (iv), $x+\delta^{\prime}$ can be taken as a point of $E$. Consequently with each $x$ of $E$ there is associated an infinite sequence of intervals $\left(x, x+\delta_{i}^{\prime}\right)$ for which (3) holds with $\delta_{i}^{\prime}$ tending to zero, and with $x+\delta_{i}^{\prime}$ points of $E$. It is, therefore, possible to select a finite non-overlapping set $\left(x_{i}, x_{i+1}\right)$ of these intervals for which (4) holds, and for which

$$
\sum\left(x_{i+1}-x_{i}\right)>d-a-\eta, \quad \eta<\delta .
$$

Let $\left(x_{j}, x_{j+1}\right)$ be the intervals on $(a, d)$ complementary to the set $\left(x_{i}, x_{i+1}\right)$. Order the intervals of these two sets from left to right into the set $\left(x_{k}, x_{k+1}\right)$. On the intervals $\left(x_{0}=a, x_{1}\right),\left(x_{l-1}, x_{l}=d\right)$, and the remaining intervals of $\left(x_{k}, x_{k+1}\right)$ which belong to the set $\left(x_{j}, x_{j+1}\right)$ let $s_{\epsilon}(x)=\left\{F\left(x_{k+1}\right)-F\left(x_{k}\right)\right\} /\left(x_{k+1}-x_{k}\right)$. On the remaining intervals of the set $\left(x_{k}, x_{k+1}\right)$ let $s_{e}(x)=F^{\prime}$, where $F^{\prime}$ is finite. Otherwise let $s_{\mathrm{e}}(x)=0$. For a fixed $k=k^{\prime}$ other than $k=0, l-1$ it follows from (1), (2), (4), and (5) that

$$
\left|F(x)-F\left(x_{k^{\prime}}\right)-\int_{x_{k^{\prime}}}^{x} s_{\epsilon} d x\right| \quad\left(x_{k^{\prime}} \leqq x \leqq x_{k^{\prime}+1}\right)
$$

is not greater than the greater of the two numbers $2 \epsilon$ and $\epsilon\left(x-x_{k^{\prime}}\right)$. We have

$$
\begin{aligned}
\int_{a}^{x} s_{\epsilon} d x & =\int_{a}^{x_{k^{\prime}}} s_{e} d x+\int_{x_{k^{\prime}}}^{x} s_{e} d x \\
& =\int_{a}^{x_{1}} s_{\epsilon} d x+\sum \int_{x_{j}}^{x_{j+1}} s_{\epsilon} d x+\sum \int_{x_{i}}^{x_{i+1}} s_{\epsilon} d x+\int_{x_{k^{\prime}}}^{x} s_{\epsilon} d x,
\end{aligned}
$$

where in each case the sum is taken over the intervals of the sets $\left(x_{i}, x_{i+1}\right)$, $\left(x_{i}, x_{i+1}\right)$ to the left of $x_{k^{\prime}}$ except $\left(x_{0}, x_{1}\right)$. It now follows from (4), (6), and the definition of $s_{e}$, that

$$
\left|F(x)-F(a)-\int_{a}^{x} s_{\epsilon} d x\right|<\epsilon(x-a)+2 \epsilon,
$$

for $x_{1} \leqq x \leqq x_{l-1}$, and for $x=d$. Also $s_{\epsilon}=F^{\prime}$ on a set $e$ with $m e>d-a-2 \eta$. This construction can be repeated for each of the intervals of the set $A_{n}$ in such a way that relation ( 7$)$ holds for each point of $(a, b)$ except possibly the points 
interior to a set of intervals $\alpha_{n}=\left(a, a+\delta_{n 0}\right),\left(d_{i}-\delta_{n i}, d_{i}\right),\left(d_{i}, d_{i}+\delta_{n i}\right)$, $\left(b-\delta_{n 0}^{\prime}, b\right)$, where $d_{i}$ represents the points of $d_{1}, \cdots, d_{n}$ other than $a$ and $b$. Furthermore $s_{e}$, now defined on $(a, b)$, is such that $s_{\epsilon}=F^{\prime}$ on a set $E_{c}$ with $m E_{\mathrm{c}}>b-a-2 n \eta$, where $\eta$ is arbitrarily small independently of $n$. If $\epsilon_{n}$ is a sequence of values of $\epsilon$ tending to zero, then for the corresponding sequence of functions $\sigma_{n}=s_{e_{n}}$ it will now be shown that

$$
F(x)-F(a)=\lim _{n \rightarrow \infty} \int_{a}^{x} \sigma_{n} d x,
$$

for all values of $x$ on $(a, b)$. Relation (7) holds for the discontinuities $d_{1}, \cdots, d_{n}$. Consequently (8) holds for all the discontinuities of $F$. There remains the consideration of points of continuity of $F$ which are on an infinite set of the open intervals $\alpha_{n}$. For a fixed $d_{i}$ the intervals $\left(d_{i}-\delta_{n i}, d_{i}\right)$, $\left(d_{i}, d_{i}+\delta_{n i}\right)$ are such that $\delta_{n i}$ tends to zero as $n$ increases. Hence if any point $x$ is on the first of these open intervals for an infinite set of values of $n$, then

$$
\begin{aligned}
\left|F(x)-F\left(d_{i}-\delta_{n i}\right)-\int_{d_{i}-\delta_{n i}}^{x} \sigma_{n} d x\right|< & \sum_{i=n+1}^{\infty}\left|s_{l}\left(d_{i}\right)\right|+\sum_{i=n+1}^{\infty}\left|s_{r}\left(d_{i}\right)\right| \\
& +\left|\psi(x)-\psi\left(d_{i}-\delta_{n i}\right)\right| .
\end{aligned}
$$

As $n$ increases each of the three terms on the right tends to zero. A similar relation holds for $x$ on $\left(d_{i}, d_{i}+\delta_{n i}\right)$. This, with the foregoing, establishes (8) for every point $x$ of $(a, b)$. The sequence $\sigma_{n}=F^{\prime}$ on $E_{n}$ where $m E_{n}$ tends to $b-a$. It then follows that there exists a subsequence $s_{n}$ of $\sigma_{n}$ and a set $\varepsilon$ with $m \varepsilon=b-a$ such that $s_{n}$ tends to $F^{\prime}$ on $\varepsilon$. Let $f(x)=F^{\prime}$ on $\varepsilon, f(x)=0$ elsewhere on $(a, b)$. Then this sequence $s_{n}$ tends to $f$ almost everywhere, and (8) holds with $s_{n}$ replacing $\sigma_{n}$. From the manner in which $s_{n}$ was constructed, it is clear that $\int_{e} s_{n} d x$ is bounded in $n$ and $e$. The function $f$ and the sequence $s_{n}$ satisfy the requirements of the theorem.

That $\int_{e} s_{n} d x$ be bounded in $n$ and $e$ is a sufficient condition for $F$ to be of bounded variation, but it is not a necessary condition. Let $x_{1}=a<x_{2}<x_{3}<\ldots$ be a sequence of values of $x$ on $(a, b)$ with $x_{n}$ tending to $b$. On the intervals $\left(x_{n-1}, x_{n}\right),\left(x_{n}, x_{n+1}\right)$ let $s_{n}$ be constant and such that the integrals of $s_{n}$ over these intervals is $n$ and $-n$ respectively. Then $F(x)=0, a \leqq x \leqq b$, but $\int_{e} s_{n}$ is not bounded in $n$ and $e$.

3. The independence of $F(x)$ and $\int_{a}^{x} f(x) d x$. Some examples are now given which show that the limit function $F(x)$ is independent of $f(x)$. We first construct a special sequence $s(a, b, r)$ on the linear interval $(a, b)$. Delete the interior points of the middle third of $(a, b)$, then the interior points of the middle third of each of the remaining thirds, and so on indefinitely. Let $G$ be 
the non-dense closed set of zero measure which remains. At the $n$th stage of this process there are $2^{n}$ undeleted intervals. On each of these intervals let $s_{n}(x)$ be constant, and such that $\int s_{n} d x$ over each interval is equal to $r / 2^{n}$, where $r$ is a prescribed real number not zero. Let $s_{n}=0$ elsewhere on $(a, b)$. If $s_{n}$ is now redefined to be zero at the points of $G$, then $\int s_{n}$ over any part of $(a, b)$ is not changed, and $s_{n}$ tends to zero everywhere. Furthermore, it is easily verified that

$$
\phi(x)=\lim _{n \rightarrow \infty} \int_{a}^{x} s_{n} d x
$$

is continuous, monotone, and $\phi(b)-\phi(a)=r$. Let this sequence $s_{n}$ be denoted by $s(a, b, r)$.

Let $F(x)$ be any continuous function on $(a, b)$. Divide $(a, b)$ into $n$ equal parts by the points $a=d_{0}, d_{1}, \cdots, d_{n}=b$. Let $F\left(d_{i}\right)-F\left(d_{i-1}\right)=r_{i}$. On $\left(d_{i-1}, d_{i}\right)$ let $s_{n}$ be the $k$ th member of the sequence $s\left(d_{i-1}, d_{i}, r_{i}\right)$, where $k$ is sufficiently great to insure that $s_{n}=0$ on a part of $(a, b)$ with measure greater than $b-a-1 / 2^{n}$. Then $\int_{a}^{d_{i}} s_{n} d x=F\left(d_{i}\right)-F(a)$. Since on $\left(d_{i}, d_{i+1}\right), \int_{d_{i}}^{x} s_{n} d x$ is monotone, for $x$ different from $d_{i}, \int_{a}^{x} s_{n} d x$ does not differ from $F(x)-F(a)$ by more than the maximum of $\left|F(x)-F\left(d_{i}\right)\right|, d_{i} \leqq x \leqq d_{i+1}$. Since this maximum tends to zero as $n$ increases, it follows that

$$
F(x)-F(a)=\lim _{n \rightarrow \infty} \int_{a}^{x} s_{n} d x .
$$

Since $s_{n}=0$ on a set $E_{n}$ with $m E_{n}>b-a-1 / 2^{n}$, it follows that there exists a set $E$ on $(a, b)$ with $m E=b-a$ at each point of which $s_{n}$ tends to zero. If $s_{n}$ is now redefined to be zero at the points of $C E$, then (1) holds with $s_{n}$ tending to zero everywhere. Now let $x_{1}, x_{2}, \cdots$ be a sequence of values of $x$ with $a<\cdots<x_{n}<x_{n-1}<\cdots$ and $x_{n}$ tending to $a$. Redefine $s_{n}$ on the interior of $\left(a, x_{n}\right)$ in such a way that $\int_{a}^{x_{n}} s_{n} d x=F(a)$. We then have

$$
F(x)=\lim _{n \rightarrow \infty} \int_{a}^{x} s_{n} d x
$$

with $s_{n}$ tending to zero everywhere. We have thus shown:

If $F(x)$ is any continuous function on $(a, b)$, then there exists $S(0, a, x)$ such that $F(x)=S(0, a, x)$, and the sequence $s_{n}$ used in defining $S(0, a, x)$ tends to sero everywhere.

Next let $F(x)$ be any measurable function on $(a, b)$. There exists a sequence of continuous functions $\phi_{n}(x)$ tending to $F(x)$ almost everywhere. By the foregoing there exists a sequence $s_{n k}$ such that $s_{n k}=0$ on a set $E_{n k}$ with $m E_{n k}>b-a-\epsilon_{k}$ and 


$$
\left|\phi_{n}(x)-\int_{a}^{x} s_{n k} d x\right|<\epsilon_{k} .
$$

The quantity $\epsilon_{k}$ is independent of $n$. Hence if $\epsilon_{k}<1 / 2^{n}$ and $s_{n}=s_{n k}$, then almost everywhere on $(a, b)$

$$
F(x)=\lim _{n \rightarrow \infty} \int_{a}^{x} s_{n} d x,
$$

and $s_{n}$ tends to zero almost everywhere. By modifying $s_{n}$ on a set of zero measure we have (3) holding with $s_{n}$ tending to zero everywhere. We thus get:

If $F(x)$ is any measurable function on $(a, b)$, then there exists $s_{n}(x)$ tending to zero everywhere for which $\int_{a}^{x} s_{n} d x$ tends to $F(x)$ almost everywhere.

Finally, let $F_{1}(x), F_{2}(x), \cdots$ be any sequence of measurable functions on $(a, b)$. There exists $s_{n k}$ tending to zero everywhere for which

$$
F_{n}(x)=\lim _{k \rightarrow \infty} \int_{a}^{x} s_{n k} d x
$$

almost everywhere. If from the double sequence $s_{n k}$ there is selected the single sequence $s_{1 k_{1}}, s_{1 k_{2}}, s_{2 k_{2}}, s_{1 k_{3}}, s_{2 k_{3}}, s_{3 k_{3}}, \ldots$ then for the single sequence $s_{n}$ obtained in this way the set of limits of $\int_{a}^{x} s_{n} d x$ includes the sequence of functions $F_{1}(x), F_{2}(x), \cdots$ for almost all points of $(a, b)$. If in defining $s_{n}$ each successive $k_{p}$ is chosen sufficiently great then $s_{n}$ tends to zero almost everywhere. By redefining this sequence $s_{n}$ at a set of zero measure we have the following:

If $F_{1}(x), F_{2}(x), \cdots$ is any sequence of measurable functions on $(a, b)$, there exists a sequence of summable functions $s_{n}(x)$ tending to zero everywhere such that for almost all points of $(a, b)$ the set of limits of $\int_{a}^{x} s_{n} d x$ includes the sequence $F_{1}(x), F_{2}(x), \cdots$.

4. The inversion of approximate derived numbers. If $f(x)$ is finite except for a denumerable set, and almost everywhere is equal to one* of the derived numbers of the continuous function $F(x)$, then $f$ is integrable in the Denjoy sense to $F(x)-F(a)$. We now obtain the corresponding theorem for approximate derived numbers, with a set of measure zero replacing the denumerable set, and sequence integration replacing Denjoy integration.

Let $f(x)$ be measurable and finite almost everywhere on $(a, b)$, and almost everywhere be equal to one of the approximate derived numbers of the continuous function $F(x)$. Since $F$ is continuous it is measurable. Then, since $f$ is finite except for a set of zero measure, it follows that $f=A D F$ almost every-

* Not necessarily the same derived number at each point. 
where. ${ }^{*}$ Let $E_{n}$ be the set for which $-n<f<n$. Then $f$ is summable over $E_{n}$, at almost all points of $E_{n}$ the density of $E_{n}$ is unity, and

$$
\lim _{h \rightarrow 0} \frac{1}{h} \int_{E_{n}(x, x+h)} f d x=f .
$$

At a point $x$ for which (1) holds let $E_{x}$ be any measurable set with right-hand density unity at $x$. Then

$$
\lim _{h \rightarrow 0} \frac{1}{h} \int_{B_{x} E_{n}(x, x+h)} f d x=f .
$$

For if $E^{\prime}=E_{n}(x, x+h)-E_{x} E_{n}(x, x+h)$, then

$$
\frac{1}{h} \int_{E_{n}(x, x+h)} f d x=\frac{1}{h} \int_{E_{x} E_{n}(x, x+h)} f d x+\frac{1}{h} \int_{E^{\prime}} f d x .
$$

Since $|f|<n$ on $E_{n}$, the second integral on the right is at most equal to $n m E^{\prime} / h$, and as $h$ tends to zero this tends to zero for the reason that the density of $E^{\prime}$ is zero at $x$. Since at almost all points of $E_{n}, f$ is the approximate derivative of $F$, we have for these points $x$,

$$
\lim _{\xi \rightarrow x} \frac{F(\xi)-F(x)}{\xi-x}=f,
$$

for a set $e_{\xi}$ of right-hand density unity at $x$. Hence, from (1) and (2), for a given $\epsilon>0$, and a given $\eta$ with $0<\eta<1$, there exists $\delta>0$ for which

$$
\left|\frac{F(\xi)-F(x)}{\xi-x}-f\right|<\frac{\epsilon}{2}, \quad\left|\frac{1}{h} \int_{\varepsilon_{\xi}(x, x+h)} f d x-f\right|<\frac{\epsilon}{2},
$$

$m e_{\xi}(x, x+h)>\eta h, 0<h<\delta, \delta$ depending on $x$, and this relation holding for almost all points of $E_{n}$. For $n$ sufficiently large $m E_{n}$ is arbitrarily near to $b-a$. We conclude, therefore, that there exists on $(a, b)$ a set $E$ with $m E=b-a$, to each point $x$ of which there corresponds a set $e_{x}$ and a number $\delta>0$ for which,

$$
\left|\frac{F(\xi)-F(x)}{\xi-x}-\frac{1}{\xi-x} \int_{0_{x}(x, \xi)} f d x\right|<\epsilon,
$$

$\xi$ belonging to $e_{x}, m e_{x}(x, x+h)>\eta h, 0<h<\delta$. Hence to each point $x$ of $E$ there corresponds a sequence of intervals $\left(x, x+h_{i}\right)$ with $h_{i}$ tending to zero

* J. C. Burkill, and U. S. Haslam-Jones, Proceedings of the London Mathematical Society, ser. 2, vol. 32 (1900), pp. 346-355. It is shown that if $F$ is measurable and finite, then almost everywhere $A D F$ exists and is finite, or $A D^{+}=A D^{-}=\infty, A D_{+}=A D_{-}=-\infty$. 
such that on $\left(x, x+h_{i}\right)$ there is a set $e_{x}$ which includes the point $x+h_{i}$, with $m e_{x}\left(x, x+h_{i}\right)>\eta h_{i}$, and for which

$$
\left|F(\xi)-F(x)-\int_{\theta_{x}(x, \xi)} f d x\right|<\epsilon(\xi-x),
$$

where on $\left(x, x+h_{i}\right)$ the set $e_{\xi}$ is the set $e_{x}\left(x, x+h_{i}\right)$. From the set of intervals thus associated with the set $E$, it is possible to select a finite set $\left(x_{k}, x_{k+1}\right)$ with $\sum\left(x_{k+1}-x_{k}\right)>m E-\epsilon$. Let $\left(x_{j}, x_{j+1}\right)$ be the intervals on $(a, b)$ complementary to the set $\left(x_{k}, x_{k+1}\right)$. Furthermore, let $\left(x_{k}, x_{k+1}\right)$ be so chosen that if $x^{\prime}$ and $x^{\prime \prime}$ are any two points on an interval of the set $\left(x_{k}, x_{k+1}\right)$ or on an interval of the set $\left(x_{i}, x_{j-1}\right)$ then

$$
\left|F\left(x^{\prime}\right)-F\left(x^{\prime \prime}\right)\right|<\epsilon .
$$

Let $s_{e \eta}=f$ on $e_{x_{k}}=e_{k}, s_{\epsilon \eta}=\left\{F\left(x_{j+1}\right)-F\left(x_{j}\right)\right\} /\left(x_{j+1}-x_{j}\right)$ on $\left(x_{j}, x_{j+1}\right)$, and $s_{\epsilon \eta}=0$ elsewhere on $(a, b)$. The function $s_{\epsilon \eta}=f$ on $\sum e_{k}$, which is a set with measure $>\eta(b-a-\epsilon)$. For $k=k^{\prime}$ and $x$ a point of $e_{k}$, we have

$$
\begin{aligned}
\left|F(x)-F(a)-\int_{a}^{x} s_{\ell \eta} d x\right| \leqq & F(x)-F\left(x_{k^{\prime}}\right)-\int_{x_{k^{\prime}}}^{x} s_{\ell \eta} d x \mid \\
& +\sum\left|F\left(x_{i+1}\right)-F\left(x_{i}\right)-\int_{x_{i}}^{x_{i+1}} s_{\ell \eta} d x\right| \\
& +\sum\left|F\left(x_{l+1}\right)-F\left(x_{l}\right)-\int_{x_{l}}^{x_{l+1}} s_{\ell \eta} d x\right|,
\end{aligned}
$$

where $\left(x_{i}, x_{i+1}\right)$ includes all the intervals of $\left(x_{k}, x_{k+1}\right)$ with $k<k^{\prime}$ and $\left(x_{l}, x_{l+1}\right)$ includes all the intervals of the set $\left(x_{i}, x_{j+1}\right)$ to the left of $x_{k^{\prime}}$. On account of (4) the first term on the right is not greater than $\epsilon\left(x-x_{k^{\prime}}\right)$, and the second term is not greater than $\epsilon\left(x_{k^{\prime}}-a\right)$. While from the definition of $s_{\epsilon \eta}$ on $\left(x_{i}, x_{i+1}\right)$, the third term is zero. Hence for $x$ any point of the set $\sum e_{k}$,

$$
\left|F(x)-F(a)-\int_{a}^{x} s_{\epsilon \eta} d x\right|<\epsilon(x-a) .
$$

Let $e_{k}^{\prime}$ be a closed subset of $e_{k}$ for which

$$
\left|\int_{e_{k}-\boldsymbol{o}_{k}^{\prime}} f d x\right|<\epsilon,
$$

and let $(\alpha, \beta)$ be an interval on $\left(x_{k}, x_{k+1}\right)$ complementary to $e_{k}^{\prime}$. If $x$ is any point on $(\alpha, \beta)$ which is not a point of $e_{k}$ then it follows from (5), (6), and (7) that 


$$
\left|F(x)-F(a)-\int_{a}^{x} s_{\ell \eta} d x\right|<\epsilon(x-a)+2 \epsilon .
$$

On an interval of the set $\left(x_{j}, x_{j+1}\right), s_{\epsilon}$ is constant. Consequently the integral of $s_{\ell \eta}$ over $\left(x_{j}, x\right)$ is linear on $\left(x_{i}, x_{i+1}\right)$ and varies from zero to $F\left(x_{j+1}\right)-F\left(x_{j}\right)$. It then follows from the relation (5) that if $x$ is a point on the interval $\left(x_{j}, x_{j+1}\right)$, we have

$$
\left|F(x)-F(a)-\int_{a}^{x} s_{\epsilon \eta} d x\right|<\epsilon(x-a)+2 \epsilon .
$$

Hence for all values of $x$ on $(a, b)$,

$$
\left|F(x)-F(a)-\int_{a}^{x} s_{\iota !} d x\right|<\epsilon(x-a)+2 \epsilon,
$$

and $s_{\iota \eta}=f$ on a set with measure $>\eta(b-a-\epsilon)$. If then we take a sequence of values of $\epsilon$ tending to zero and a corresponding sequence of values of $\eta$ tending to unity, we arrive at a sequence of functions, $s_{n}$, for which

$$
F(x)-F(a)=\lim _{n \rightarrow \infty} \int_{a}^{x} s_{n} d x .
$$

Furthermore, since $s_{n}=f$ on a set with measure $>\eta_{n}\left(b-a-\epsilon_{n}\right)$ it follows that there exists a subsequence of $s_{n}$ which converges to $f$ almost everywhere on $(a, b)$. We have thus proved

TheOREM V. Let the function $f(x)$ be measurable and finite almost everywhere on $(a, b)$, and almost everywhere be one or the other of the approximate derived numbers of the continuous function $F(x)$. Then $f(x)$ is integrable in the sequence sense to $F(x)-F(a)$.

5. The limit of $s_{n}(x)$ not summable. Let $x_{1}, x_{2}, \cdots$ be a sequence of values of $x$ on $(0,1)$ with $x_{1}=0, x_{i}<x_{i+1}$, and with $x_{i}$ tending to unity. On $\left(x_{i}, x_{i+1}\right)$ let $f= \pm 1 /\left[i\left(x_{i+1}-x_{i}\right)\right]$, + or - holding accordingly as $i$ is odd or even. Let $s_{n}=f$ on $\left(x_{i}, x_{i+1}\right), i=1,2, \cdots, n$, and $s_{n}=0$ elsewhere. Then the integral of $f$ exists on $(0,1)$ as a non-absolutely convergent integral, $s_{n}$ is summable for each $n$, and

$$
\int_{0}^{x} f(x) d x=F(x)=\lim _{n \rightarrow \infty} \int_{0}^{x} s_{n}(x) d x, \quad 0 \leqq x \leqq 1 .
$$

Let $\delta_{i}=\left(x_{i}, x_{i+1}\right)$. On $\delta_{i}$ the function $f$ is positive or negative accordingly as $i$ is odd or even. Let the sequence $\delta_{1}, \delta_{2}, \cdots$ be rearranged in the order $\delta_{1}, \delta_{3}, \delta_{2}, \delta_{5}, \delta_{7}, \delta_{4}, \cdots$, two intervals on which $f$ is positive followed by 
one on which $f$ is negative. Let this rearranged sequence be $\gamma_{1}, \gamma_{2}, \cdots$, let $s_{n}=f$ on $E_{n}=\gamma_{1}, \gamma_{2}, \cdots, \gamma_{n}$, and let $s_{n}=0$ elsewhere on $(0,1)$. Then

(2) $\lim _{n \rightarrow \infty} \int_{0}^{x} s_{n} d x=\int_{0}^{x} f(x) d x, \quad 0 \leqq x<1 ; \quad \lim _{n \rightarrow \infty} \int_{0}^{1} s_{n} d x>\int_{0}^{1} f d x$.

In both (1) and (2) $s_{n}=f$ on $E_{n}, E_{n} \supset E_{n-1}$, and $m E_{n}$ tends to unity. We thus see that for the function $f$ defined above there is a sequence $s_{n}$ of this general type for which (1) holds, and another such sequence for which (1) does not hold. This raises the question: If $f$ is any function which is integrable in a non-absolutely convergent sense, does there exist at least one sequence of this general type for which (1) holds? The answer is in the affirmative for the generalized Denjoy integral. The proof of this is built up in several stages.

Let $f(x)$ be a measurable function which is integrable in the generalized Denjoy sense on $(a, b)$. Let $E_{1}$ be the points of non-summability of $f$ on $(a, b)$, and $\left(\alpha_{i}, \beta_{i}\right)$ the set of open intervals complementary to $E_{1}$. Fix $\epsilon_{n}$ and let $\left(\alpha_{n}^{\prime}, \beta_{n}^{\prime}\right)$ be an interval with $\alpha_{i}<\alpha_{n}^{\prime}<\beta_{n}^{\prime}<\beta_{i}$, and such that for $x$ on $\left(\alpha_{i}, \alpha_{n}^{\prime}\right)$, $\left(\beta_{n}^{\prime}, \beta_{i}\right)$ we have respectively

$$
\left|F(x)-F\left(\alpha_{i}\right)\right|<\epsilon_{n}, \quad\left|F(x) .-F\left(\beta_{i}\right)\right|<\epsilon_{n} .
$$

The function $f$ is summable on $\left(\alpha_{n}^{\prime}, \beta_{n}^{\prime}\right)$, and if $s_{n}=f$ on $\left(\alpha_{n}^{\prime}, \beta_{n}^{\prime}\right)$ and $s_{n}=0$ elsewhere on $\left(\alpha_{i}, \beta_{i}\right)$, then

$$
\left|F(x)-F\left(\alpha_{i}\right)-\int_{\alpha_{i}}^{x} s_{n} d x\right|<2 \epsilon_{n}, \quad \alpha_{i} \leqq x \leqq \beta_{i} .
$$

Letting $\epsilon_{n}$ tend to zero and $\alpha_{n}^{\prime}, \beta_{n}^{\prime}$ tend monotonically to $\alpha_{i}, \beta_{i}$ respectively we get

$$
F(x)-F\left(\alpha_{i}\right)=\lim _{n \rightarrow \infty} \int_{\alpha_{i}}^{x} s_{n} d x, \quad \alpha_{i} \leqq x \leqq \beta_{i},
$$

where $s_{n}=f$ on $E_{n}, s_{n}=0$ elsewhere on $\left(\alpha_{i}, \beta_{i}\right), E_{n} \supset E_{n-1}$, and $m E_{n}$ tends to $\beta_{i}-\alpha_{i}$. Hence we have:

On each interval $\left(\alpha_{i}, \beta_{i}\right)$ complementary to $E_{1}$ there exists $T S(f, a, x)$ such that $F(x)-F\left(\alpha_{i}\right)=T S(f, a, x)$.

Let $E_{2}$ be the points of non-summability of $f$ over $E_{1}$ together with the points of $E_{1}$ at which $\sum\left|F\left(\beta_{i}\right)-F\left(\alpha_{i}\right)\right|$ diverges, $\left(\alpha_{i}, \beta_{i}\right)$ the intervals complementary to $E_{1}$. Now let $\left(\alpha_{i}, \beta_{i}\right)$ be an interval of the set complementary to $E_{2}$. Fix $\epsilon_{n}$ and let $\left(\alpha_{n}^{\prime}, \beta_{n}^{\prime}\right)$ be an interval with $\alpha_{i}<\alpha_{n}^{\prime}<\beta_{n}^{\prime}<\beta_{i}$ such that for $x$ on $\left(\alpha_{i}, \alpha_{n}^{\prime}\right),\left(\beta_{n}^{\prime}, \beta_{i}\right)$ we have respectively 


$$
\left|F(x)-F\left(\alpha_{i}\right)\right|<\epsilon_{n}, \quad\left|F(x)-F\left(\beta_{i}\right)\right|<\epsilon_{n} .
$$

Let $e$ be the part of $E_{1}$ on $\left(\alpha_{n}^{\prime}, \beta_{n}^{\prime}\right),\left(\alpha_{j}, \beta_{j}\right)$ the intervals complementary to $e$ on $\left(\alpha_{n}^{\prime}, \beta_{n}^{\prime}\right)$. Then $\sum\left|F\left(\beta_{j}\right)-F\left(\alpha_{j}\right)\right|$ converges. Fix $p_{n}$ so that

$$
\sum_{j=p_{n}+1}^{\infty}\left|F\left(\beta_{j}\right)-F\left(\alpha_{j}\right)\right|<\epsilon_{n},
$$

and so that for $j>p_{n}$ and $x$ on $\left(\alpha_{j}, \beta_{j}\right)$,

$$
\left|F(x)-F\left(\alpha_{j}\right)\right|<\epsilon_{n} .
$$

At a point $x$ of $e$ on $\left(\alpha_{n}^{\prime}, \beta_{n}^{\prime}\right)$ we have

$$
F(x)-F\left(\alpha_{n}^{\prime}\right)=\sum_{\left(\alpha_{n}^{\prime}, x\right)}\left\{F\left(\beta_{j}\right)-F\left(\alpha_{j}\right)\right\}+\int_{o\left(\alpha_{n}^{\prime}, x\right)} f d x .
$$

On $\left(\alpha_{i}, \beta_{i}\right)\left(j=1,2, \cdots, p_{n}\right)$ there exists $E_{n j}$ with $m E_{n j}$ arbitrarily near to $\left(\alpha_{i}, \beta_{j}\right)$, and $s_{n j}=f$ on $E_{n i}, s_{n j}=0$ elsewhere on $\left(\alpha_{j}, \beta_{j}\right)$ for which

$$
\left|F(x)-F\left(\alpha_{j}\right)-\int_{\alpha_{j}}^{x} s_{n j} d x\right|<\frac{\epsilon_{n}}{p_{n}}, \quad \alpha_{j} \leqq x \leqq \beta_{j} .
$$

If $s_{n}=s_{n j}$ on $\left(\alpha_{i}, \beta_{j}\right), s_{n}=f$ on $e$, and $s_{n}=0$ elsewhere on $\left(\alpha_{i}, \beta_{i}\right)$, it follows from (1), (2), (3), (4), and (5) that

$$
\left|F(x)-F\left(\alpha_{i}\right)-\int_{\alpha_{i}}^{x} s_{n} d x\right|<4 \epsilon_{n} .
$$

Let $\epsilon_{n}$ tend to zero, $\alpha_{n}^{\prime}, \beta_{n}^{\prime}$ tend monotonically to $\alpha_{i}, \beta_{i}$ respectively, and $p_{n}$ increase monotonically. Also on the intervals $\left(\alpha_{j}, \beta_{j}\right), j=1,2, \cdots, p_{n}$, let $E_{n j}$ be so determined that $E_{n j}$ contains $E_{(n-1) j}$ and $\sum_{j} m E_{n j}$ tends to $\sum\left(\beta_{j}-\alpha_{j}\right)$. Then

$$
F(x)-F\left(\alpha_{i}\right)=\lim _{n \rightarrow \infty} \int_{\alpha_{i}}^{x} s_{n} d x, \quad \alpha_{i} \leqq x \leqq \beta_{i},
$$

$s_{n}=f$ on $E_{n}, E_{n} \supset E_{n-1}$ and $m E_{n}$ tends to $\beta_{i}-\alpha_{i}$. Thus we have:

On all the intervals $\left(\alpha_{i}, \beta_{i}\right)$ complementary to $E_{2}$ there exists $T S(f, a, x)$ for which $F(x)-F\left(\alpha_{i}\right)=T S(f, a, x), \alpha_{i} \leqq x \leqq \beta_{i}$.

If $E_{3}$ is the set of points of non-summability of $f$ over $E_{2}$ together with the points of $E_{2}$ at which $\sum\left|F\left(\beta_{i}\right)-F\left(\alpha_{i}\right)\right|$ diverges, $\left(\alpha_{i}, \beta_{i}\right)$ the intervals complementary to $E_{2}$, then the foregoing process can be repeated to obtain the corresponding result for the intervals $\left(\alpha_{i}, \beta_{i}\right)$ complementary to $E_{3}$. Furthermore, the process can be repeated for every set $E_{\lambda}$ for $\lambda<\omega$, where $\omega$ is the 
first transfinite ordinal of the second kind. Let $\left(\alpha_{i}, \beta_{i}\right)$ be an interval of the set complementary to $E_{\omega}$ and $\left(\alpha_{n}^{\prime}, \beta_{n}^{\prime}\right)$ an interval with $\alpha_{i}<\alpha_{n}^{\prime}<\beta_{n}^{\prime}<\beta_{i}$ and with

$$
\left|F(x)-F\left(\alpha_{i}\right)\right|<\epsilon_{n}, \quad\left|F(x)-F\left(\beta_{i}\right)\right|<\epsilon_{n},
$$

for $x$ on $\left(\alpha_{i}, \alpha_{n}^{\prime}\right),\left(\beta_{n}^{\prime}, \beta_{i}\right)$ respectively. There is a set $E_{\lambda}, \lambda<\omega$ for which the part of $E_{\lambda}$ on $\left(\alpha_{n}^{\prime}, \beta_{n}^{\prime}\right)$ is empty. It then follows that the methods of construction given above lead to the existence of $T S\left(f, \alpha_{n}^{\prime}, x\right)$, for which $T S\left(f, \alpha_{n}^{\prime}, x\right)$ $=F(x)-F\left(\alpha_{n}^{\prime}\right), \alpha_{n}^{\prime} \leqq x \leqq \beta_{n}^{\prime}$. Hence on $\left(\alpha_{n}^{\prime}, \beta_{n}^{\prime}\right)$ there exists $s_{n k}=f$ on $E_{n k}$, $s_{n k}=0$ elsewhere on $\left(\alpha_{n}^{\prime}, \beta_{n}^{\prime}\right), m E_{n k}>\beta_{n}-\alpha_{n}-\epsilon_{n}$, and

$$
\left|F(x)-F\left(\alpha_{n}^{\prime}\right)-\int_{\alpha_{n}}^{x} s_{n k} d x\right|<\epsilon_{n}, \quad \alpha_{n}^{\prime} \leqq x \leqq \beta_{n}^{\prime} .
$$

If $\epsilon_{n}$ tends to zero and $\alpha_{n}^{\prime}, \beta_{n}^{\prime}$ tend respectively to $\alpha_{i}, \beta_{i}$, it follows from (6) and (7) that, if $s_{n k}=s_{n}$, then

$$
F(x)-F\left(\alpha_{i}\right)=\lim _{n \rightarrow \infty} \int_{\alpha_{i}}^{x} s_{n} d x, \quad \alpha_{i} \leqq x \leqq \beta_{i},
$$

where $s_{n}=f$ on $E_{n}, s_{n}=0$ elsewhere on the interval $\left(\alpha_{i}, \beta_{i}\right)$ and $m E_{n}$ tends to $\beta_{i}-\alpha_{i}$. Let $\epsilon_{1}, \epsilon_{2}, \cdots$ be a sequence of values of $\epsilon$ tending to zero. Fix $n_{1}$ so that

$$
\left|F(x)-F\left(\alpha_{i}\right)-\int_{\alpha_{1}}^{x} s_{n_{1}} d x\right|<\epsilon_{1}, \quad \alpha_{i} \leqq x \leqq \beta_{i} .
$$

Let $\varepsilon_{1}=E_{n_{1}}$ and $\sigma_{1}=s_{n_{1}}$. Fix $n_{2}$ so that

$$
\left|F(x)-F\left(\alpha_{i}\right)-\int_{\alpha_{i}}^{x} s_{n_{2}} d x\right|<\epsilon_{2}, \quad \alpha_{i} \leqq x \leqq \beta_{i},
$$

and so that

$$
\int_{G}|f| d x<\epsilon_{2}
$$

where $G=\varepsilon_{1}-E_{n_{2}}$. Relation (10) is possible for the reason that $f$ is summable on $\varepsilon_{1}$ and $m E_{n}$ tends to $\left(\beta_{i}-\alpha_{i}\right)$. Set $\varepsilon_{2}=E_{n_{2}}+\left(\varepsilon_{1}-\varepsilon_{n_{2}}\right)$. Then $\varepsilon_{2} \supset \varepsilon_{1}$. Also, if $\sigma_{2}=f$ on $\varepsilon_{2}, \sigma_{2}=0$ elsewhere on $\left(\alpha_{i}, \beta_{i}\right)$ it follows from (9) and (10) that

$$
\left|F(x)-F\left(\alpha_{i}\right)-\int_{\alpha_{i}}^{x} \sigma_{2} d x\right|<2 \epsilon_{2}, \quad \alpha_{i} \leqq x \leqq \beta_{i} .
$$

This process can be repeated indefinitely, giving a sequence of functions $\sigma_{1}, \sigma_{2}, \cdots$ for which 


$$
F(x)-F\left(\alpha_{i}\right)=\lim _{n \rightarrow \infty} \int_{\alpha_{i}}^{x} \sigma_{n} d x,
$$

where $\sigma_{n}=f$ on $\varepsilon_{n}, \sigma_{n}=0$ elsewhere on $\left(\alpha_{i}, \beta_{i}\right), \varepsilon_{n} \supset \varepsilon_{n-1}$ and $m \varepsilon_{n}$ tends to $\beta_{i}-\alpha_{i}$. This allows us to state:

If $\omega$ is the first transfinite ordinal of the second kind and $\left(\alpha_{i}, \beta_{i}\right)$ an interval on $(a, b)$ complementary to $E_{\omega}$, then there exists $T S\left(f, \alpha_{i}, x\right)$ for which $T S\left(f, \alpha_{i}, x\right)=F(x)-F\left(\alpha_{i}\right), \alpha_{i} \leqq x \leqq \beta_{i}$.

The processes of construction given above can be repeated to give the corresponding result for an interval $\left(\alpha_{i}, \beta_{i}\right)$ of the set complementary to $E_{\lambda}$, where $\lambda$ is any finite or transfinite ordinal of the first kind, or for an interval $\left(\alpha_{i}, \beta_{i}\right)$ of the set complementary to $E_{\omega}$, where $\omega$ is any transfinite ordinal of the second kind. The method of transfinite induction can now be used to prove:

THEOREM VI. If $f(x)$ is measurable on $(a, b)$ and integrable in the generalized Denjoy sense to $F(x)$, then there exists $\operatorname{TS}(f, a, x)$ for which $T S(f, a, x)$ $=F(x), a \leqq x \leqq b$.

The sequence $s_{1}, s_{2}, \cdots$ of Theorem $\mathrm{V}$ converges to $f$ almost everywhere, but is not defined wholly in terms of $f$. There may be intervals $\left(x_{j}, x_{i+1}\right)$ on $(a, b)$ for which $s_{n}=\left\{F\left(x_{j+1}\right)-F\left(x_{j}\right)\right\} /\left(x_{j+1}-x_{j}\right)$, and consequently $s_{n}$ cannot be determined on these intervals without a knowledge of the values of $F$ at the points $x_{j}, x_{j+1}$. In some cases $s_{n}$ can be determined without a knowledge of the values of $F$ at particular points. To throw further light on this point we start with a continuous function $F(x)$ which is also $(A C G)$, and prove

LEMMA I. Let $F(x)$ be $(A C G)$ on $(a, b)$, and let $e$ be any closed set with $m e=0$. Then there exists a finite set of intervals $\left(a_{n}, b_{n}\right)$ with $\left(a_{n}, b_{n}\right)$ points of $e$ which contain, either as end points or interior points, all of e except at most a finite set, and for which

$$
\sum\left|F\left(b_{n}\right)-F\left(a_{n}\right)\right|<\epsilon, \quad \sum\left(b_{n}-a_{n}\right)<\delta,
$$

where $\epsilon$ and $\delta$ are arbitrary positive numbers.

Under the conditions of the lemma $e=e_{1}+e_{2}+\cdots$, where $F$ is absolutely continuous on each $e_{n}$. Let $\bar{e}_{n}$ be the set $e_{n}$ together with its limit points. Then, since $e$ is closed, $\bar{e}_{n} \subset e$, and the continuity of $F$ can be used to show that $F$ is absolutely continuous on $\bar{e}_{n}$. Let $\left(\alpha_{i}, \beta_{i}\right)$ be the intervals on $(a, b)$ contiguous to $\bar{e}_{1}$, and $\left(c_{1 i}^{\prime}, c_{1 i^{\prime}}\right)$ the finite set of intervals on $(a, b)$ belonging to the complement of the intervals $\left(\alpha_{1}, \beta_{1}\right), \cdots,\left(\alpha_{n}, \beta_{n}\right)$. Then the intervals $\left(c_{1 i}^{\prime}, c_{1 i}^{\prime \prime}\right)$ contain, either as end points or interior points, all of $\bar{e}_{1}$, except at most a finite set. The points $c_{i}^{\prime}, c_{i}^{\prime \prime}$ belong to $\bar{e}_{1}$. Then, since $F$ is 
absolutely continuous on $\bar{e}_{1}$ and since $m \bar{e}_{1}=0$, it follows that if $n$ is sufficiently great,

$$
\sum\left|F\left(c_{1 i}^{\prime \prime}\right)-F\left(c_{1 i}^{\prime}\right)\right|<\epsilon_{1}, \quad \sum\left(c_{1 i}^{\prime \prime}-c_{1 i}^{\prime}\right)<\delta_{1} .
$$

Let $B_{1}^{\prime}, B_{2}^{\prime}, \cdots$ be the finite set of intervals complementary to the set $\left(c_{1 i}^{\prime}, c_{1 i}^{\prime \prime}\right)$, and let $\bar{e}_{2 i}$ be the part of $\bar{e}_{2}$ on the closed interval $B_{i}^{\prime}$. There then exists on $B_{i}^{\prime}$ a finite set of intervals containing, either as end points or interior points, all of the set $\bar{e}_{2 i}$, except at most a finite number of points of $\bar{e}_{2 i}$, and which satisfy relations similar to (1). If $\left(c_{2}^{\prime}, c_{2 i}^{\prime \prime}\right)$ is the total set of intervals thus determined for all of the set $B_{i}^{\prime}$, it is possible to have

$$
\sum\left|F\left(c_{2 i}^{\prime \prime}\right)-F\left(c_{2 i}^{\prime}\right)\right|<\epsilon_{2}, \quad \sum\left(c_{2 i}^{\prime \prime}-c_{2 i}^{\prime}\right)<\delta_{2} .
$$

The set $\left(c_{1 i}^{\prime}, c_{1 i}^{\prime \prime}\right)+\left(c_{2 i}^{\prime}, c_{2 i}^{\prime \prime}\right)$ contains all of $\bar{e}_{1}$ and $\bar{e}_{2}$, except for at most a finite number of points. We designate by $B_{i}^{2}$ the finite set of intervals on $(a, b)$ complementary to $\left(c_{1 i}^{\prime}, c_{1 i}^{\prime \prime}\right)+\left(c_{2 i}^{\prime}, c_{2 i}^{\prime \prime}\right)$, and proceed to determine on $B_{i}^{2}$ a finite set of intervals containing all of $\bar{e}_{3 i}$, except at most a finite number of points. Continuing this process we arrive at the countable set of intervals, $\left(c_{j i}^{\prime}, c_{j i}^{\prime \prime}\right)$ none of which overlap, which contain, either as end points or as interior points, all of $e$ except at most a countable set $P$, and for which, if $\sum \epsilon_{i}<\epsilon, \sum \delta_{i}<\delta$,

$$
\sum_{i} \sum_{i}\left|F\left(c_{j i}^{\prime \prime}\right)-F\left(c_{j i}^{\prime}\right)\right|<\epsilon, \quad \sum_{i} \sum_{i}\left(c_{j i}^{\prime \prime}-c_{j i}^{\prime}\right)<\delta .
$$

Let $D$ be the end points of the intervals $C=\left(c_{j i}^{\prime}, c_{j i}^{\prime \prime}\right)$, and $E$ the limit points of the set $D+P$. The set $D+P$ is enumerable. Since $e$ is closed and $C+P \supset e$, it follows that $D+P \supset E$. Consequently $E$ is enumerable and closed. Let $E=x_{1}, x_{2}, \cdots$. Since $e$ is closed and $m e=0$, the set $e$ is non-dense on $(a, b)$. Hence each point $x_{i}$ of the set $E$ is the left-hand end point of an interval $\left(x_{i}, x_{i}^{\prime}\right)$, or the right-hand end point of an interval $\left(x_{i}^{\prime}, x_{i}\right)$, or both, where in the first case $x_{i}^{\prime}$ is a point of $e$ which is not a limit point of $e$ on the right, and in the second case $x_{i}^{\prime}$ is a point of $e$ which is not a limit point of $e$ on the left. Thus $E$ is on a set of intervals whose end points are not limit points of $e$ from without. Furthermore, these intervals can be chosen so that

$$
\left|F\left(x_{i}^{\prime}\right)-F\left(x_{i}\right)\right|<\epsilon_{i}, \quad\left|x_{i}^{\prime}-x_{i}\right|<\delta_{i} .
$$

Since $E$ is closed, it easily follows that there exists a finite non-overlapping set $A$ of these intervals associated with the set $E=x_{1}, x_{2}, \ldots$ which contain, either as end points or interior points, all the points of $E$. If an end point of the set $A$ happens to be an interior point of an interval $\left(c_{i j}^{\prime}, c_{i j}{ }^{\prime}\right)$, it can be changed to one or the other of the end points of $\left(c_{i j}^{\prime}, c_{i j}{ }^{\prime \prime}\right)$ without altering relations (4). There will then be only a finite set $C^{\prime}$ of the intervals of $C$ 
which are exterior to or abutting the altered set $A$, and if $\left(a_{n}, b_{n}\right)$ is the finite set of intervals $C^{\prime}+A$, then $\left(a_{n}, b_{n}\right)$ contains, either as interior points or end points, all of $e$ except at most a finite set, the points $a_{n}, b_{n}$ belong to $e$, and

$$
\sum\left|F\left(b_{n}\right)-F\left(a_{n}\right)\right|<3 \epsilon, \quad \sum\left(b_{n}-a_{n}\right)<3 \delta .
$$

This establishes the lemma.

Let the function $f$ be finite almost everywhere on $(a, b)$, and almost everywhere be equal to one or the other of the approximate derived numbers of the function $F$ which is $(A C G)$ on $(a, b)$. Working as in Theorem $\mathrm{V}$, it is possible to determine on $(a, b)$ a finite set of intervals $\left(x_{k}, x_{k+1}\right)$, and on each interval a set $e_{k}$, where

$$
\left|F(\xi)-F\left(x_{k}\right)-\int_{e_{k}\left(x_{k}, \xi\right)} f d x\right|<\epsilon\left(\xi-x_{k}\right),
$$

where the set $\xi$ is the set $e_{k}$, where $m e_{k}>\eta\left(x_{k+1}-x_{k}\right)$, and where $\sum\left(x_{k+1}-x_{k}\right)$ is arbitrarily near to $b-a$, in particular $>(b-a) / 2$. Denote this finite set of intervals by $K_{1}$ and the part of $(a, b)$ complementary to $K_{1}$ by $C K_{1}$. It is then possible to determine on $C K_{1}$ a finite set of intervals $K_{2}$ satisfying relations similar to (5), with $m K_{2}>m C K_{1} / 2$. Denoting the part of $(a, b)$ complementary to $K_{1}+K_{2}$ by $C K_{2}$, we can determine a set $K_{3}$ on $C K_{2}$ with $m K_{3}>m C K_{2} / 2$, and with the set $K_{3}$ satisfying relations similar to (5). Continuing this process we arrive at a set of non-overlapping intervals $K=K_{1}+K_{2}+\cdots$ with $m K=b-a$. Let $e$ be the end points and limit points of end points of $K$. Then $e$ is closed, $m e=0$, and consequently, this set $e$ satisfies the conditions of Lemma $I$ in relation to $F$. Let $\left(a_{n}^{\prime}, b_{n}^{\prime}\right)$ be the set of intervals provided by this lemma. It is evident that these intervals can be reduced to a non-abutting set $\left(a_{n}, b_{n}\right)$ with the same properties relatively to $F$. The complement of the closed intervals $\left(a_{n}, b_{n}\right)$ and the finite number of points of $e$ exterior to $\left(a_{n}, b_{n}\right)$ is a finite set of open intervals $\left(x_{k}, x_{k+1}\right)$ of the set $K$. Let $s_{\epsilon \eta}=f$ on $e_{k}, s_{\epsilon \eta}=0$ elsewhere on $(a, b)$. Then, as in Theorem V, for any point of $(a, b)$,

$$
\begin{aligned}
\left|F(x)-F(a)-\int_{a}^{x} s_{\epsilon \eta} d x\right| & <\epsilon(x-a)+2 \epsilon+\sum\left|F\left(b_{n}\right)-F\left(a_{n}\right)\right| \\
& <\epsilon(b-a)+3 \epsilon .
\end{aligned}
$$

Also $\sum m e_{k}>\eta \sum\left(x_{k+1}-x_{k}\right)>\eta(b-a-\epsilon)$. If now we take a sequence of values of $\epsilon$ tending to zero, and a corresponding sequence of values of $\eta$ tending to unity, we arrive at a sequence of summable functions $s_{n}$ for which $s_{n}=f$ on a set $E_{n}, s_{n}=0$ elsewhere, $m E_{n}$ tends to $b-a$, and 


$$
F(x)-F(a)=\lim _{n \rightarrow \infty} \int_{a}^{x} s_{n}^{\prime} d x .
$$

Proceeding as in the concluding part of the proof of Theorem VI, a subsequence $\sigma_{n}$ of $s_{n}$ can be determined for which (6) holds and for which $\sigma_{n}=f$ on $\varepsilon_{n}, \sigma_{n}=0$ elsewhere, $\varepsilon_{n} \supset \varepsilon_{n-1}$, and $m \varepsilon_{n}$ tends to $b-a$. Thus we have proved

TheOREM VII. Let $f(x)$ be finite almost everywhere on $(a, b)$, and almost everywhere be equal to one or the other of the approximate derived numbers of the continuous function $F(x)$ which is also $(A C G)$. Then $f(x)$ is totally integrable in the sequence sense to $F(x)-F(a)$.

If $f(x)$ satisfies the conditions of Theorem VII it is integrable in the generalized Denjoy sense.* Hence Theorem VII follows from Theorem VI. Conversely Theorem VI follows from Theorem VII. For if $F(x)$ is the generalized Denjoy integral of $f$ then $F$ is $(A C G)$, and almost everywhere $A D F=f$. Between the proofs of these two theorems there are these distinctions: Theorem VII holds for continuous functions $F(x)$ which are not $(A C G)$, but which behave relatively to every closed set of zero measure in the manner described by Lemma I, provided such functions exist. Again the proof of Theorem VII does not involve transfinite induction, and gives, therefore, a method for constructing a generalized Denjoy integral without the use of transfinite numbers. This construction is particularly simple when the points $E$ of non-summability of $f$ are of zero measure.

The set $E$ is closed. It then follows from Lemma $I$ that there is a finite set $\left(a_{n}, b_{n}\right)$ of non-abutting intervals containing all of $E$ except a finite set $P$, with $\sum\left|F\left(b_{n}\right)-F\left(a_{n}\right)\right|<\epsilon$. Let $\left(\alpha_{i}, \beta_{i}\right)$ be the finite number of intervals complementary to the set $\left(a_{n}, b_{n}\right)+P$. If $\left(\alpha_{i}{ }^{e}, \beta_{i}{ }^{e}\right)$ is an interval with $\alpha_{i}<\alpha_{i}{ }^{e}$ $<\beta_{i} i<\beta_{i}$, then on this interval $f$ is summable. Furthermore, if, for each $i$, $\alpha_{i}{ }^{e}, \beta_{i}{ }^{\epsilon}$ are sufficiently near to $\alpha_{i}, \beta_{i}$ respectively, then

$$
\left|F(b)-F(a)-\sum \int_{a_{i}^{a}}^{\beta_{i}^{\epsilon}} f d x\right|<2 \epsilon,
$$

and $F(b)-F(a)$ is obtained by taking a sequence of values of $\epsilon$ tending to zero.

6. Conditions for uniqueness of total sequence integration. We first construct a function $T S(f, a, x)$ which is not equal to $\int_{a}^{x} f(x) d x$. Let $G$ be the Cantor non-dense closed set on $(0,1)$ defined as in $\S 3$. Let $(a, b)$ be the middle third of $(0,1), x_{0}=a<x_{1}<x_{2}<\cdots$, a sequence of values of $x$ on $(a, b)$ with

* Saks, loc. cit., p. 197, §2. 
$x_{n}$ tending to $b$. On $\left(x_{i-1}, x_{i}\right), i \neq 1$, let $f(x)= \pm 1 /\left[i\left(x_{i}-x_{i-1}\right)\right],+$ or - holding accordingly as $i$ is odd or even. On $\left(x_{0}, x_{1}\right)$ let $f$ be constant and such that $\int_{x_{0}}^{x_{1}} f d x=1-\log 2$. Then $\int_{a}^{x} f d x$ exists as a Denjoy integral on $(a, b)$, with $b$ the single point of non-summability of $f$, and this integral has the value zero at $x=b$. Let $\left(a^{\prime}, b^{\prime}\right)$ be one of the two intervals deleted from $(0,1)$ in the second step in the construction of $G$. Let the point $x^{\prime}$ on $a^{\prime} \leqq x^{\prime} \leqq b^{\prime}$ correspond to the point $x$ on $a \leqq x \leqq b$ by means of a one-to-one projective transformation which carries $a^{\prime}$ into $a$ and $b^{\prime}$ into $b$. At $x^{\prime}$ on $\left(a^{\prime}, b^{\prime}\right)$ let $f\left(x^{\prime}\right)=f(x) / 2^{2}$, where $x$ is the point on $(a, b)$ which corresponds to $x^{\prime}$ on $\left(a^{\prime}, b^{\prime}\right)$. The function $f\left(x^{\prime}\right)$ is integrable in the Denjoy sense on $\left(a^{\prime}, b^{\prime}\right), b^{\prime}$ is the single point of non-summability of $f\left(x^{\prime}\right)$, and

$$
\int_{a}^{x^{\prime}} f\left(x^{\prime}\right) d x^{\prime}=\frac{1}{2} \cdot \frac{1}{2} \int_{a}^{x} f(x) d x
$$

$x$ and $x^{\prime}$ corresponding points on $\left(a^{\prime}, b^{\prime}\right)$ and $(a, b)$. On each $\left(a^{\prime}, b^{\prime}\right)$ of the four deleted intervals arising in the third step of the construction of $G$ let $f\left(x^{\prime}\right)$ be defined similarly, except that $f\left(x^{\prime}\right)=f(x) / 2^{4}$, which gives

$$
\int_{a^{\prime}}^{x^{\prime}} f\left(x^{\prime}\right) d x^{\prime}=\frac{1}{4} \cdot \frac{1}{2^{2}} \int_{a}^{x} f(x) d x
$$

where $x$ and $x^{\prime}$ are corresponding points. Continuing this process, and setting $f=0$ on $G$, the function $f$ is integrable in the Denjoy sense on $(0,1)$. If $\left(\alpha_{i}, \beta_{i}\right)$ are the intervals complementary to $G, F(x)=\int_{a}^{x} f d x$ is such that $F\left(\alpha_{i}\right)=F\left(\beta_{i}\right)=0$, and for $x$ a point interior to $\left(\alpha_{i}, \beta_{i}\right), F(x)=\int_{\alpha_{1}}^{x} f d x$.

Let $\lambda$ be a fixed positive number $>1-\log 2$, and on $(a, b)$, the middle third of $(0,1)$, let $e_{1}, e_{2}, \cdots, e_{n}$ be the intervals $\left(x_{0}, x_{1}\right),\left(x_{2}, x_{3}\right), \cdots$, $\left(x_{2 n}, x_{2 n+1}\right)$. On these intervals $f$ is positive. There exists $n$ such that

$$
\sum_{i=1}^{n-1} \int_{e_{i}} f d x<\lambda, \quad \sum_{i=1}^{n} \int_{e_{i}} f d x \geqq \lambda .
$$

Let $E_{11}=e_{1}+e_{2}+\cdots+e_{n}$. Let $\delta_{21}, \delta_{22}, \delta_{23}$ be the three deleted intervals arising at the end of the second stage in the construction of $G$, ordered from left to right. On $\delta_{22}$ the middle third of $(a, b)$ let $E_{22}=\left(x_{0}, x_{2 n}\right)+e_{n+1}+\cdots$ $+e_{n+p}$, where $e_{n+1}=\left(x_{2 n+2}, x_{2 n+3}\right), e_{n+2}=\left(x_{2 n+4}, x_{2 n+5}\right), \cdots$ and where

$$
\int_{a}^{x_{2 n}} f d x+\sum_{i=1}^{p-1} \int_{e_{n+i}} f d x<\frac{\lambda}{3}, \quad \int_{a}^{x_{2 n}} f d x+\sum_{i=1}^{p} \int_{e_{n+i}} f d x \geqq \frac{\lambda}{3} .
$$

On $\delta_{21}, \delta_{23}$ obtain sets $E_{21}, E_{23}$ by repeating the construction of the set $E_{11}$ on $(a, b)$ with $\lambda / 3$ replacing $\lambda$. Let $\varepsilon_{1}=E_{11}, \varepsilon_{2}=\sum E_{2 i}$. Then $\varepsilon_{2}$ contains $\varepsilon_{1}$. Let $\delta_{31}, \delta_{32}, \cdots, \delta_{37}$ be the seven deleted intervals arising at the end of the 
third step in the construction of $G$. On $\delta_{3 i}$ construct the set $E_{3 i}$ according to the above scheme with $\lambda / 7$ replacing $\lambda / 3$, taking care that $\varepsilon_{3}=\sum E_{3 i}$ contains $\varepsilon_{2}$. This process of construction can be continued, giving the sets $\varepsilon_{1}, \varepsilon_{2}, \ldots$ with $\varepsilon_{n} \supset \varepsilon_{n-1}$ and $m \varepsilon_{n}$ tending to unity. Let $s_{n}=f$ on $\varepsilon_{n}$ and $s_{n}=0$ elsewhere on $(0,1)$. Then $s_{n}$ is summable on $(0,1), s_{n}$ tends to $f$, and it is easily verified that

$$
\lim _{n \rightarrow \infty} \int_{a}^{x} s_{n} d x=\phi(x),
$$

where $\phi(x)$ is continuous. Let $x$ be any point on $(0,1)$, and let $R_{n}(x)$ be the number of whole deleted intervals to the left of $x$ arising at the $n$th step in the construction of $G$. There are $2^{n}-1$ of these intervals, and it is easily verified that as $n$ increases $R_{n}(x) /\left(2^{n}-1\right)$ tends to a limit $R(x)$, where $R(x)$ is continuous, non-decreasing, constant on the intervals $\left(\alpha_{i}, \beta_{i}\right)$ complementary to $G$, and such that $R(0)=0, R(1)=1$. Furthermore

$$
\phi(x)=\lambda R(x)+\int_{a}^{x} f d x .
$$

We thus have $\phi(x)=T S(f, a, x) \neq \int_{a}^{x} f d x$.

The function $\phi(x)$ is not $(A C G)$ on $(0,1)$. Suppose the contrary to be true. If $\phi$ is $(A C G)$ on $(0,1)$ then, since $G$ is closed and $m G=0$, it follows from Lemma $I$ that there exists a finite set of intervals $\left(a_{n}, b_{n}\right)$ on $(0,1)$ containing all of $G$ except a finite set $P$, where $a_{n}, b_{n}$ are points of $G, \sum\left(b_{n}-a_{n}\right)$ is arbitrarily small, the intervals $\left(\gamma_{n}, \delta_{n}\right)$ complementary to $\left(a_{n}, b_{n}\right)+P$ are a finite number of the intervals $\left(\alpha_{i}, \beta_{i}\right)$ complementary to $G$, and the sum $\sum\left|\phi\left(b_{n}\right)-\phi\left(a_{n}\right)\right|$ is arbitrarily small. Since $R(x)$ is constant on $\left(\alpha_{i}, \beta_{i}\right)$, it follows from (2) that $\phi\left(\beta_{i}\right)-\phi\left(\alpha_{i}\right)=\int_{\alpha_{i}}^{\beta_{i}} f d x=0$. Hence $\phi\left(\delta_{n}\right)-\phi\left(\gamma_{n}\right)=0$. But

$$
\phi(1)-\phi(0)=\sum\left\{\phi\left(b_{n}\right)-\phi\left(a_{n}\right)\right\}+\sum\left\{\phi\left(\delta_{n}\right)-\phi\left(\lambda_{n}\right)\right\} \text {. }
$$

Since the first term on the right can be made arbitrarily small by a proper choice of the intervals $\left(a_{n}, b_{n}\right)$ and since the second term on the right is zero for every choice of $\left(a_{n}, b_{n}\right)$ in accordance with the requirements of Lemma I, it follows that $\phi(1)-\phi(0)=0$. Again, since $R(0)=0, R(1)=1$, and $\int_{0}^{1} f d x=0$, it follows from (2) that $\phi(1)-\phi(0)=1$. We thus get a contradiction, and are able to conclude that $\phi(x)$ is not $(A C G)$ on $(a, b)$.

It is possible to prove:

THEOREM VIII. If $f$ is integrable in the generalized Denjoy sense on $(a, b)$, if the set $E$ of points of non-summability of $f$ has zero measure, and if $T S(f, a, x)$ exists which is $(A C G)$, then

$$
T S(f, a, x)=\int_{a}^{x} f d x=F(x) .
$$


Since $m E=0$ we can consider that $f=0$ on $E$. There exists an interval $(l, m)$ containing a part $e$ of $E$ on its interior such that if $\left(\alpha_{i}, \beta_{i}\right)$ are the intervals on $(l, m)$ complementary to $E$, then $\sum\left\{F\left(\beta_{i}\right)-F\left(\alpha_{i}\right)\right\}$ converges. If $\left(\alpha^{\prime}, \beta^{\prime}\right)$ is an interval such that $\alpha_{i}<\alpha^{\prime}<\beta^{\prime}<\beta_{i}$, then $f$ is summable on $\left(\alpha^{\prime}, \beta^{\prime}\right)$ and consequently $T S\left(f, \alpha^{\prime}, \beta^{\prime}\right)=F\left(\beta^{\prime}\right)-F\left(\alpha^{\prime}\right)$. It then follows from the continuity of $T S(f, a, x)$ that $T S\left(f, \alpha_{i}, \beta_{i}\right)=F\left(\beta_{i}\right)-F\left(\alpha_{i}\right)$. Let $\phi(x)$ $=T S(f, a, x)$ and apply Lemma $I$ as above to get $\left(a_{n}, b_{n}\right)$ and $\left(\gamma_{n}, \delta_{n}\right)$ with $\left(\gamma_{n}, \delta_{n}\right)$ an interval of the set $\left(\alpha_{i}, \beta_{i}\right)$ and consequently $\phi\left(\gamma_{n}\right)-\phi\left(\delta_{n}\right)=F\left(\delta_{n}\right)$ $-F\left(\gamma_{n}\right)$, and $\sum\left|\phi\left(b_{n}\right)-\phi\left(a_{n}\right)\right|$ arbitrarily small. These intervals can be so chosen that $\sum\left\{F\left(\delta_{n}\right)-F\left(\gamma_{n}\right)\right\}$ is at the same time arbitrarily near to $F(m)-F(l)$. It follows from these considerations that $\phi(m)-\phi(l)=F(m)$ $-F(l)$, and for $x$ on $(l, m), \phi(x)-\phi(l)=F(x)-F(l)$. The method of transfinite induction can now be used to show that for $x$ on $(a, b), \phi(x)=T S(f, a, x)$ $=F(x)$.

Added in proof, December 9, 1936. It is now known that there exists $T S(f, a, x)$ which is $(A C G)$ with $T S(f, a, x) \neq \int_{a}^{x} f d x$. The proof of this, together with necessary and sufficient conditions for $T S(f, a, x)=\int_{a}^{x} f d x$ will be published in a subsequent paper.

Acadia University,

Wolfville, Nova Scotia, aNd

The University of Wisconsin, Madison, Wis. 